\title{
Health Differences across the Three Obesity Classes: Evidence from the 2012 Behavioral Risk Factor Surveillance System
}

\author{
Lauren Needell and Ari Mwachofi* \\ Department of Public Health, East Carolina University, USA
}

Submission: April 22, 2017; Published: July 28, 2017

*Corresponding author: Ari Mwachofi, Department of Public Health, Brody School of Medicine, East Carolina University, USA, Tel: 252-744-5073; Email: mwachofia@ecu.edu

Abstract

Obesity increases the burden of disease, decreases the quality of life and life expectancy, and contributes over $\$ 200$ billion annually to US health expenditures. Although there are three distinct obesity classes, most studies lump them together and examine obesity as one condition. Analysis of health effects of the three obesity classes could provide leads to more targeted and insightful interventions.

Objectives: The study questions are: Are there health differences in self-assessed health across the three obesity classes? Are there differences in prevalence of diagnosed chronic health conditions in these obesity classes?

Method: We address the study questions through analysis of data from the 2012 Behavioral Risk Factor Surveillance System (BRFSS) of the US Centers for Disease Control and Prevention (CDC).We define six body-weight groups: BMI $\leq 18.5$ as underweight; healthy body weight as18.5 $\leq \mathrm{BMI}<25$; overweight as $25<\mathrm{BMI}<30$; Class1 obeseas $30 \leq \mathrm{BMI}<35$; Class2 obese as $35 \leq \mathrm{BMI}<40$; and Class 3 obese as $40 \leq \mathrm{BMI}$. We conduct $\chi 2$ and t-tests of differences in self-assessed health status and prevalence of diagnosed chronic health conditions in the three obesity classes. Applying a health production framework from health economics, and using overweight and healthy body-weight as the control group, we conduct multivariate analysis of the effects of obesity on self-assessed general and physical health.

Result: There are significant $(\mathrm{p}<0.000)$ differences in self-assessed health status, and in prevalence of diagnosed chronic health conditions across these obesity classes. The higher the obesity class the poorer the health. Class 3 has the largest negative effect on the likelihood of good general and physical health.

Conclusion: Health differences across obesity classes suggest the need to examine obesity in greater detail. Rather than addressing obesity as a single problem, it might be more helpful to examine levels of obesity and to tailor interventions to specific body-weight classes.

Keywords: Health status; Body weight classes; Severe obesity; Morbid obesity; Class 1 obese; Class 2 obese; Class 3 obese; Health production; Health production inputs self-assessed health; Chronic health conditions; Body mass index (BMI)

\section{Introduction}

Obesity is one of the most costly public health problems in the world. In 2008, an estimated 502 million adults were obese [1]. On average in the US, obese individuals die 9.44 years earlier than those not obese [2], which equals more than 125 million years of potential life lost due to obesity [3]. An estimated 111,909 extra deaths occur among obese people compared to deaths among individuals with healthy body weights [4]. Between 2011and 2012, 78.6 million people in the United States (34.9\% of adults) were obese [5]. Annually in the US, 3.4 million quality-adjusted life years are lost to obese women and 1.94 million to obese men [6]. Obesity not only has serious physical health implications, but also has serious mental health consequences $[7,8]$. Estimates of obesity-attributable excess medical expenditures amount to $\$ 147$ billion annually while productivity losses amount to $\$ 66$ billion [9].

Obesity exacerbates over twenty major chronic health conditions [10]. It is both a primary and secondary risk factor for coronary heart disease and is positively correlated with the prevalence and severity [11-16]. Some evidence suggests that obesity affects strokes [17-19] and asthma is a well-documented co morbidity for obesity [20-23]. People with chronic obstructive pulmonary disease (COPD) have higher prevalence of obesity and 
obesity in COPD patients is associated with significantly more severe activity limitations and increased health care utilization [24]. However, a closer examination of COPD conditions shows that patients with chronic bronchitis are more likely to be obese while those with emphysema are more likely to be underweight [25].

Obesity is related to numerous cancers. Esophageal adenocarcinoma is aggravated by obesity through reflux esophagi is and chronic irritation [26]. Obesity-related inflammation leads to multiple myeloma and Non-Hodgkin's lymphoma [27], and might result in chronic kidney disease [28]. Obesity is also linked to renal cancer [29], to colon, pancreatic and liver cancers $[30,31]$ and to gallbladder cancer [32]. In women, obesity is linked to endometrial and pre- and postmenopausal breast cancers [33]. Other obesity-related cancers include thyroid, rectal, leukemia, prostate and malignant melanoma [34]. Among individuals diagnosed with cancer, those who are obese have decreased survivorship [35].

Obesity research mostly combines all obesity groups together and focuses on prevalence, but not on obesity variations and their differential effects on health [36-38]. In consideration of obesity heterogeneity and the scope of management options, three classes are defined as Class 1including BMI from 30 to less than 35; Class 2 also known as severe obesity, including BMI of 35 to less than 40; and Class 3also known as morbid obesity, including BMI that is equal to or greater than 40 [39].

\section{Study Objectives}

The main study question is: Are there health differences across the three obesity classes? Related questions are: Are there differences in prevalence of chronic health conditions in these obesity classes? Relative to individuals with healthy body weight, what is the health status of individuals in the three obesity classes?

\section{Study Methods}

\section{Study model}

The study applies a household health production framework from health economics, which posits that the household produces health using household, individual and environmental inputs [39]. Some health production inputs (e.g. meals, shelter) are produced by the household. The basic model used in previous studies [40-42], can be represented by the following health production function:

$$
\mathrm{Hi}=\mathrm{f}(\mathrm{Ii}, \mathrm{E},)
$$

Where: the subscript $\mathrm{i}$ denotes the individual as the unit of analysis; $\mathrm{H}$ is a vector depicting health output; $\mathrm{I}$ is a set of individual and household variables (inputs) and E represents environmental inputs. Researchers have applied this framework in studies of various health-related phenomena such as effects of prenatal care on birth weights [43]. Household production and demand for health inputs and their effects on birth weights [44], Effects of childhood and education on health [45], the impact of maternal smoking on child neuro development [46] and the relationship between household production, fertility and child mortality [47].

Within the health production framework, obesity Classes 1, 2 and 3 are individual variable inputs in health production. These classes might also be representative of health behavior (such as diet and exercise) or descriptive of health capital stock [40]. Based on the household health production process represented by equation 1 above the econometric model used in multivariate analysis of general health $(\mathrm{GH})$, and physical health $(\mathrm{PH})$ has the following two equations:

$$
\begin{aligned}
& \mathrm{GHi}=\mathrm{f}(\mathrm{Di}, \mathrm{Si} \text { Bi,Hi Ei }) . \\
& \text { PHi= f( Di,Si Bi,Hi Ei) }
\end{aligned}
$$

Where: D represents demographic factors; $\mathrm{S}$ is socioeconomic status (SES); B is health behaviors; $\mathrm{H}$ is health capital stock, $\mathrm{E}$ are environmental factors such as access to care. These equations were utilized in multivariate analysis examining the effects of the three obesity classes on health.

Health is measured as

i. Self-assessed general health status

ii. Self-assessed physical health status, and

iii. Number of poor health days experienced within a 30day period.

\section{Data source and study sariables}

The study data are from the 2012 Behavioral Risk Factor Surveillance System (BRFSS) survey. BRFSS is an annual nationwide telephone survey of non-institutionalized adults. The survey is conducted by the CDC in collaboration with health departments in all states [48]. The survey is based on a multistage cluster design that uses random-digit dialing to select samples that are representative of the US population.

Dependent variables: The 2012 BRFSS survey had questions about individual self-assessed general health (GH) status: Would you say that in general your health is 1. Excellent, 2.Very good, 3. Good, 4. Fair, or 5. Poor? Responses to this question were coded one (1) for excellent, very good or good health and zero (0) for fair or poor health. Other BRFSS questions quantify poor health experiences in number of days of poor health within a 30-day period: Now thinking about your physical health, which includes physical illness and injury, for how many days during the past 30 days was your physical health not good? Responses to these questions provided quantitative measures of the individuals' experiences of poor health.

Independent variables: Data about $\mathrm{BMI}$, the variable of interest, were derived from responses to two BRFSS questions: About how much do you weigh without shoes? About how tall 
are you without shoes? Responses to these questions were used to calculate respondents' body mass index (BMI), which was then coded into six weight classes: $\mathrm{BMI}<18.5$ is underweight; $18.5 \leq \mathrm{BMI}<25$ is healthy weight; $25 \leq \mathrm{BMI}<30$ is overweight; $30 \leq \mathrm{BMI}<35$ is Class 1 obese; $35 \leq \mathrm{BMI}<40$ is Class 2 and $\mathrm{BMI} \geq 40$ is Class 3 obese.

Other questions gathered data about demographics (age, ethnicity, sex/gender, race, marital and veteran status) and socioeconomic status (SES) such as income, employment, home-ownership, educational levels and access to personal cell-phones. Other questions were used as surrogate measures of household climate. These include number of dependent children, if the household is female headed with no adult males or if it is male headed with no adult females. Measures of individual health behavior include tobacco and alcohol use, physical exercise, the use of seatbelts in automobiles, getting vaccinations, and health screenings such as HIV-tests. BRFSS also provided data about access to care and health capital stock. Access to care was measured using three variables: having health insurance and personal doctors and inability to access care due to high costs of care. Individual stock of health capital was measured as disability status and diagnosed chronic health conditions. The two measures of disability used responses to BRFSS questions: Are you limited in any way in any activities because of physical, mental, or emotional problems? Do you now have any health problem that requires you to use special equipment, such as a cane, a wheelchair, a special bed, or a special telephone? Responses to these questions were coded one (1) for "yes" and zero (0) for "no." Data about diagnosed chronic health conditions were derived from responses to BRFSS survey question: Has a doctor, nurse, or other health professional EVER told you that you had any of the following: heart attack also called a myocardial infarction, angina or coronary heart disease, stroke, asthma, skin cancer, other types of cancer, chronic

Table 1: Summary statistics and variable definitions. obstructive pulmonary disease (COPD), arthritis, depressive disorder, kidney disease, trouble seeing, diabetes? Responses to these questions were coded one (1) for "yes" and zero (0) for "no."It is important to note that BRFSS defines arthritis to include rheumatoid arthritis, gout, lupus, or fibromyalgia and COPD to include emphysema or chronic bronchitis.

\section{Analysis Methods}

We use dt- and $\chi 2$ tests for statistical significance of health differences across the obesity classes. We used $\chi 2$ testsfor categorical variables and t-tests on differences in the number of days of poor health within a 30-day period. We conducted three sets of tests: differences betweenClasses 1 and 2, Classes 1 and 3 and differences between class 2 and 3 .

Multivariate analysis estimated the likelihood of good health as represented in equations 2 and 3above, and enabled the study to measure the effects of the three obesity classes (relative to the control group) while controlling for other health production factors. In estimating the differential effects of the three obesity classes, the study used normal and overweight groups as the controls. Table 1 displays summary statistics of the study sample, which is drawn from all states in the US. It includes health condition, obesity class and the variable definitions applied in the study. The obesity class with the largest number of respondents is Class 1 while Class 2 had the smallest number. As expected, individuals who are obese have worse health than those with healthy body weights. The proportion of individuals with healthy body weights who were diagnosed with chronic health conditions is much lower than proportions in the three obese classes. Those who are obese also experience more days of poor health than those with healthy body weights. They also have lower proportions with self-assessed good or excellent health status.

\begin{tabular}{|c|c|c|c|c|c|c|}
\hline Health Condition & Sample \% & $\begin{array}{c}\text { Healthy-Weight } \\
\%\end{array}$ & Obesity Class & \% OR Mean & Std. Dev & $\mathbf{N}$ \\
\hline \multirow{3}{*}{$\begin{array}{c}\text { Self-assessed } \\
\text { General Health } \\
\text { Status } \\
\text { (good to excellent } \\
=1 \text { fair or poor }=0 \text { ) }\end{array}$} & \multirow[b]{3}{*}{80.4} & \multirow[b]{3}{*}{85.4} & One & 76.3 & 0.43 & 79266 \\
\hline & & & Two & 68.2 & 0.47 & 29245 \\
\hline & & & Three & 55.5 & 0.50 & 18297 \\
\hline \multirow{3}{*}{$\begin{array}{c}\text { Self-assessed } \\
\text { Physical Health } \\
\text { Status } \\
\text { (good to excellent } \\
=1 \text { fair or poor }=0 \text { ) }\end{array}$} & \multirow{3}{*}{61.8} & \multirow{3}{*}{65.9} & One & 57.96 & 0.43 & 79838 \\
\hline & & & Two & 50.5 & 0.50 & 29429 \\
\hline & & & Three & 40.7 & 0.49 & 18388 \\
\hline \multirow{3}{*}{$\begin{array}{l}\text { Heart attack } \\
\text { (if had a heart } \\
\text { attack=1 } \\
\text { otherwise }=0 \text { ) }\end{array}$} & 6.2 & 4.7 & One & 7.5 & 0.26 & 79169 \\
\hline & & & Two & 8.1 & 0.27 & 29201 \\
\hline & & & Three & 8.5 & 0.28 & 18216 \\
\hline
\end{tabular}




\section{Current Research in Diabetes \& Obesity Journal}

\begin{tabular}{|c|c|c|c|c|c|c|}
\hline \multirow{3}{*}{$\begin{array}{c}\text { Angina (if } \\
\text { had angina }=1 \\
\text { otherwise }=0 \text { ) }\end{array}$} & \multirow{3}{*}{6.4} & \multirow{3}{*}{4.6} & One & 7.8 & 0.27 & 78770 \\
\hline & & & Two & 8.9 & 0.28 & 29008 \\
\hline & & & Three & 10.0 & 0.30 & 18121 \\
\hline \multirow{3}{*}{$\begin{array}{l}\text { Stroke (if had } \\
\text { a stroke }=1 \\
\text { otherwise }=0 \text { ) }\end{array}$} & \multirow{3}{*}{4.1} & \multirow{3}{*}{3.6} & One & 4.5 & 0.21 & 79297 \\
\hline & & & Two & 5.1 & 0.22 & 29235 \\
\hline & & & Three & 5.4 & 0.23 & 18290 \\
\hline \multirow{3}{*}{$\begin{array}{c}\text { Asthma (if } \\
\text { ever diagnosed } \\
\text { with asthma=1 } \\
\text { otherwise }=0 \text { ) }\end{array}$} & \multirow{3}{*}{12.7} & \multirow{3}{*}{10.6} & One & 14.5 & 0.35 & 79267 \\
\hline & & & Two & 19.4 & 0.40 & 29207 \\
\hline & & & Three & 26.0 & 0.44 & 18272 \\
\hline \multirow{3}{*}{$\begin{array}{c}\text { Skin cancer (if } \\
\text { had skin cancer }=1 \\
\text { otherwise }=0 \text { ) }\end{array}$} & \multirow{3}{*}{8.7} & \multirow{3}{*}{9.2} & One & 7.9 & 0.27 & 79351 \\
\hline & & & Two & 7.0 & 0.26 & 29253 \\
\hline & & & Three & 5.2 & 0.22 & 18300 \\
\hline \multirow{3}{*}{$\begin{array}{l}\text { Other cancer } \\
\text { (if had other } \\
\text { cancer=1 } \\
\text { otherwise }=0 \text { ) }\end{array}$} & \multirow{3}{*}{9.2} & \multirow{3}{*}{9.0} & One & 9.4 & 0.29 & 79345 \\
\hline & & & Two & 9.1 & 0.29 & 29259 \\
\hline & & & Three & 9.2 & 0.29 & 18306 \\
\hline \multirow{3}{*}{$\begin{array}{c}\text { COPD (if } \\
\text { has COPD }=1 \\
\text { otherwise }=0 \text { ) }\end{array}$} & \multirow{3}{*}{8.1} & \multirow{3}{*}{6.8} & One & 9.0 & 0.29 & 79094 \\
\hline & & & Two & 11.9 & 0.32 & 29129 \\
\hline & & & Three & 16.7 & 0.37 & 18191 \\
\hline \multirow{3}{*}{$\begin{array}{c}\text { Arthritis (if } \\
\text { has arthritis=1 } \\
\text { otherwise }=0 \text { ) }\end{array}$} & \multirow{3}{*}{34.5} & \multirow{3}{*}{27.5} & One & 41.3 & 0.49 & 79139 \\
\hline & & & Two & 47.1 & 0.50 & 29183 \\
\hline & & & Three & 54.0 & 0.50 & 18267 \\
\hline \multirow{3}{*}{$\begin{array}{l}\text { Kidney disease (if } \\
\text { diagnosed with } \\
\text { kidney disease }=1 \\
\text { otherwise }=0 \text { ) }\end{array}$} & \multirow{3}{*}{3.3} & \multirow{3}{*}{2.6} & One & 3.8 & 0.19 & 79247 \\
\hline & & & Two & 5.1 & 0.22 & 29193 \\
\hline & & & Three & 6.0 & 0.24 & 18243 \\
\hline \multirow{3}{*}{$\begin{array}{c}\text { Diabetes(if } \\
\text { diagnosed with } \\
\text { diabetes }=1 \\
\text { otherwise }=0 \text { ) }\end{array}$} & \multirow{3}{*}{12.5} & \multirow{3}{*}{5.5} & One & 19.1 & 0.39 & 79391 \\
\hline & & & Two & 26.9 & 0.44 & 29273 \\
\hline & & & Three & 34.8 & 0.48 & 18299 \\
\hline \multirow{3}{*}{$\begin{array}{l}\text { Number of poor } \\
\text { general health } \\
\text { days in a 30-day } \\
\text { period (Mean) }\end{array}$} & \multirow{3}{*}{5.69} & & One & 5.11 & 9.48 & 77895 \\
\hline & & 4.4 & Two & 6.48 & 10.36 & 28725 \\
\hline & & & Three & 8.91 & 11.66 & 17924 \\
\hline Number of poor & & & One & 5.1 & 9.48 & 78204 \\
\hline $\begin{array}{l}\text { physical health } \\
\text { davs in a 30-dav }\end{array}$ & 5.69 & 3.57 & Two & 6.48 & 10.36 & 28831 \\
\hline period (Mean) & & & Three & 8.91 & 11.66 & 17968 \\
\hline
\end{tabular}

These data also show that the higher the obesity class, the poorer the health. For example individuals with obesity Class 1 have better general health (greater percentage with excellent to good health and fewer number of poor health days) than those in the other two classes. Individuals who are Class 2 obese have slightly better health than the Class 3 obese. This is also true for diagnosis of all chronic conditions except for cancer. The trend for skin and other cancer diagnosis is opposite. Class 1 obese has greater proportions diagnosed with these two conditions than Class 2 or Class 3 obese but lower proportions than individuals with healthy body weights.
Results of t-tests of differences in number of days that respondents experienced poor health within a 30-day period are displayed in Table 2 . The higher the obesity class, the greater the number of poor health days. Individuals with Class 1 obesity experience 1.38 days less of poor physical health than individuals with Class 2 and 3.81 days less than those with Class 3. Class 1 individuals also experience 1.08 days less of poor mental health than those with Class 2 and 2.68 days less than those with Class 3 obesity. Furthermore, individuals with Class 2 obesity have fewer days of poor physical (2.43 days less) and mental health (1.6 days less) than individuals with Class 3 obesity. All the differences are statistically significant $(\mathrm{p}<0.000)$. 


\section{Current Research in Diabetes \& Obesity Journal}

Table 2: T-tests of differences across the three obesity classes, in the number of days in poor physical health in a 30-day period.

\begin{tabular}{|c|c|c|c|c|c|c|}
\hline & Mean Difference & \multicolumn{2}{|c|}{$\mathbf{9 5 \% C I}$ of difference } & \multirow{2}{*}{ t-statistic } & \multirow{2}{*}{ Sig } & \multirow{2}{*}{ N } \\
\cline { 2 - 5 } & & Lower & Upper & & \\
\hline Obese1 \& Obese 2 & -1.38 & -1.51 & -1.25 & -20.53 & .000 & 107033 \\
\hline Obese1 \& Obese3 & -3.81 & -3.97 & -3.64 & -46.35 & .000 & 96170 \\
\hline Obese 2 \& Obese 3 & -2.43 & -2.23 & -2.63 & -23.49 & .000 & 46797 \\
\hline
\end{tabular}

\section{Differences in self-assessed health status and in diagnosed chronic conditions}

Table 3: $\mathrm{X}^{2}$-tests of health and chronic condition differences across the three obesity classes.

\begin{tabular}{|c|c|c|c|c|c|c|c|c|c|}
\hline \multirow{2}{*}{$\begin{array}{l}\text { Health } \\
\text { Status }\end{array}$} & \multicolumn{3}{|c|}{ Class $1 \& 2$} & \multicolumn{3}{|c|}{ Class $1 \& 3$} & \multicolumn{3}{|c|}{ Class $1 \& 3$} \\
\hline & $\chi^{2}$ & sig & $\mathbf{N}$ & $\chi^{2}$ & sig & $\mathbf{N}$ & $\chi^{2}$ & sig & $\mathbf{N}$ \\
\hline $\begin{array}{l}\text { Good } \\
\text { General } \\
\text { Health }\end{array}$ & 744.84 & .000 & 108940 & 3228.57 & .000 & 97926 & 775.80 & .000 & 47694 \\
\hline $\begin{array}{c}\text { Good } \\
\text { Physical } \\
\text { Health }\end{array}$ & 483.01 & .000 & 109267 & 1801.17 & .000 & 98226 & 440.14 & .000 & 47817 \\
\hline \multicolumn{10}{|c|}{ Diagnosed Condition } \\
\hline $\begin{array}{l}\text { Heart } \\
\text { Attack }\end{array}$ & 12.94 & .000 & 108370 & 20.78 & .000 & 97385 & $1.73 \mathrm{~s}$ & .189 & 47417 \\
\hline Angina & 30.13 & .000 & 107778 & 91.98 & .000 & 96891 & 17.287 & .000 & 47129 \\
\hline Stroke & 15.3 & .000 & 108532 & 26.38 & .000 & 97587 & $2.42 \mathrm{~s}$ & .120 & 47525 \\
\hline COPD & 208.19 & .000 & 108223 & 947.67 & .000 & 97285 & 219.59 & .000 & 47320 \\
\hline Diabetes & 794.93 & .000 & 108664 & 2149.44 & .000 & 97690 & 332.81 & .000 & 47572 \\
\hline $\begin{array}{l}\text { Kidney } \\
\text { Disease }\end{array}$ & 92.19 & .000 & 108440 & 169.93 & .000 & 97490 & 15.29 & .000 & 47436 \\
\hline Asthma & 381.69 & .000 & 108474 & 1396.17 & .000 & 97539 & 281.28 & .000 & 47479 \\
\hline Arthritis & 291.43 & .000 & 108322 & 966.03 & .000 & 97406 & 212.03 & .000 & 47450 \\
\hline Skin Cancer & 23.28 & .000 & 108604 & 154.22 & .000 & 97651 & 60.67 & .000 & 47553 \\
\hline $\begin{array}{c}\text { Other } \\
\text { Cancers }\end{array}$ & $3.15^{s}$ & .076 & 108604 & $0.58^{\mathrm{s}}$ & .445 & 97651 & $0.395^{s}$ & .529 & 47565 \\
\hline
\end{tabular}

Table 3 displays results of $\chi 2$-test of differences in selfassessed general and physical health and diagnosed chronic conditions. Similar to differences in number of days of poor health, individuals with Class1 obesity have better self-assessed general health and lower proportions diagnosed chronic conditions than those in the higher obesity classes.

As indicated by the $\chi 2$ statistics, all differences between Classes 1 and 2 obese are statistically significant except for proportions diagnosed with other cancers. The differences in this diagnosis are small and statistically insignificant. The greatest and most significant differences between Classes 1 and 2 is self-assessed general and physical health and in proportions diagnosed with diabetes, asthma, arthritis and COPD.

As noted earlier, the greatest differences are between individuals with obesity Classes 1 and 3. All differences between these two classes are statistically significant except for proportions diagnosed with other cancers. This difference is small and statistically insignificant. The most significant differences between Classes 1 and 3 are in self-assessed general and physical health and in proportions diagnosed with diabetes, asthma, arthritis and COPD. Differences between Classes 2 and 3 are less pronounced and some are statistically insignificant. These include differences in proportions diagnosed with heart attack, stroke, and other cancers. The most pronounced differences between Classes 2 and 3 obese are in self-assessed general and physical health and the proportions diagnosed with diabetes, asthma, COPD and arthritis.

\section{Multivariate analysis result}

The control group for this analysis was individuals with BMI ranging between 18.5 and less than 25 (18.5 $\geq$ BMI < 25). This BMI range includes individuals with healthy weights and the over-weight group. This analysis included the underweight class $(\mathrm{BMI}<18.5)$ as an explanatory variable. Other explanatory variables are demographics (gender age, ethnicity), household climate, weight/obesity class, socioeconomic status, individual health behavior (smoking, drinking, physical exercise, seat-belt use, taking necessary tests/screenings, and taking necessary vaccination), access to care and health capital stock measured in terms of diagnosed chronic health conditions. Multivariate analysis results appear in Tables $4 \& 5$. 


\section{Current Research in Diabetes \& Obesity Journal}

Table 4: Effects of obesity levels on the likelihood of good general health.

\begin{tabular}{|c|c|c|c|c|c|c|c|}
\hline \multirow{2}{*}{ Demographics } & \multirow{2}{*}{ B } & \multirow{2}{*}{ S.E. } & \multirow{2}{*}{ Wald } & \multirow{2}{*}{ Sig. } & \multirow{2}{*}{$\begin{array}{c}\operatorname{Exp}(B)(\text { Odds- } \\
\text { ratio) }\end{array}$} & \multicolumn{2}{|c|}{ 95\% C.I.for EXP(B) } \\
\hline & & & & & & Lower & Upper \\
\hline Female & .205 & .019 & 111.403 & .000 & 1.227 & 1.181 & 1.274 \\
\hline Latino & -.750 & .025 & 876.537 & .000 & .473 & .450 & .497 \\
\hline Young & .190 & .031 & 37.348 & .000 & 1.209 & 1.138 & 1.285 \\
\hline Veteran & .018 & .021 & .764 & .382 & 1.018 & .978 & 1.060 \\
\hline \multicolumn{8}{|c|}{ Household Climate } \\
\hline $\begin{array}{l}\text { Dependent } \\
\text { Children }\end{array}$ & .025 & .008 & 9.020 & .003 & 1.025 & 1.009 & 1.042 \\
\hline $\begin{array}{l}\text { No Adult } \\
\text { Women }\end{array}$ & -.008 & .022 & .134 & .714 & .992 & .950 & 1.036 \\
\hline No Adult Men & .076 & .018 & 18.421 & .000 & 1.078 & 1.042 & 1.116 \\
\hline \multicolumn{8}{|l|}{ Weight/Obesity } \\
\hline Underweight & -.422 & .048 & 78.664 & .000 & .656 & .597 & .720 \\
\hline Obese class 1 & -.079 & .016 & 23.180 & .000 & .924 & .895 & .954 \\
\hline Obese class 2 & -.258 & .023 & 124.151 & .000 & .773 & .738 & .809 \\
\hline Obese class 3 & -.469 & .028 & 283.023 & .000 & .626 & .593 & .661 \\
\hline \multicolumn{8}{|l|}{ SES } \\
\hline Unemployed & -.549 & .018 & 908.067 & .000 & .578 & .557 & .599 \\
\hline Education Level & .277 & .007 & 1619.134 & .000 & 1.319 & 1.302 & 1.337 \\
\hline Income & .116 & .004 & 913.777 & .000 & 1.123 & 1.115 & 1.132 \\
\hline $\begin{array}{l}\text { Has personal } \\
\text { Cellphone }\end{array}$ & .250 & .015 & 265.778 & .000 & 1.283 & 1.246 & 1.323 \\
\hline \multicolumn{8}{|c|}{ Health Behavior } \\
\hline Current-drinker & .317 & .014 & 510.815 & .000 & 1.373 & 1.335 & 1.411 \\
\hline $\begin{array}{l}\text { Pneumonia } \\
\text { shot }\end{array}$ & -.130 & .014 & 82.833 & .000 & .878 & .854 & .903 \\
\hline Physical Ex. & .551 & .014 & 1614.952 & .000 & 1.735 & 1.689 & 1.783 \\
\hline Seat belt always & .043 & .018 & 5.779 & .016 & 1.044 & 1.008 & 1.081 \\
\hline HIV-tested & -.073 & .016 & 21.665 & .000 & .930 & .901 & .959 \\
\hline \multicolumn{8}{|l|}{ Access to Care } \\
\hline Insured & .037 & .024 & 2.493 & .114 & 1.038 & .991 & 1.088 \\
\hline $\begin{array}{c}\text { Has personal } \\
\text { doctor }\end{array}$ & -.088 & .024 & 13.707 & .000 & .916 & .875 & .960 \\
\hline Cost-Barred & -.451 & .020 & 498.888 & .000 & .637 & .612 & .663 \\
\hline \multicolumn{8}{|c|}{ Health Capital } \\
\hline Heart Attack & -.449 & .024 & 348.896 & .000 & .639 & .609 & .669 \\
\hline Angina & -.637 & .023 & 756.635 & .000 & .529 & .505 & .553 \\
\hline Stroke & -.372 & .026 & 197.198 & .000 & .689 & .654 & .726 \\
\hline Asthma & -.163 & .019 & 74.934 & .000 & .849 & .818 & .881 \\
\hline Other cancer & -.551 & .019 & 885.270 & .000 & .576 & .556 & .597 \\
\hline COPD & -.672 & .020 & 1101.029 & .000 & .511 & .491 & .532 \\
\hline Arthritis & -.377 & .014 & 722.944 & .000 & .686 & .667 & .705 \\
\hline Depression & -.340 & .016 & 468.316 & .000 & .712 & .690 & .734 \\
\hline Kidney disease & -.630 & .029 & 478.086 & .000 & .532 & .503 & .563 \\
\hline
\end{tabular}




\section{Current Research in Diabetes \& Obesity Journal}

\begin{tabular}{|c|c|c|c|c|c|c|c|}
\hline Poor Sight & -.411 & .015 & 730.264 & .000 & .663 & .644 & .683 \\
\hline Diabetes & -.729 & .016 & 1977.307 & .000 & .482 & .467 & .498 \\
\hline Disability & -1.125 & .015 & 5802.094 & .000 & .325 & .315 & .334 \\
\hline $\begin{array}{c}\text { Assistive } \\
\text { Devices }\end{array}$ & -.553 & .018 & 990.782 & .000 & .575 & .556 & .596 \\
\hline Model fit & $\mathrm{R}^{2}=.466$ & \multicolumn{2}{|c|}{$\chi^{2}=87333.31$} & Accurate Prediction $=86.2 \%$ & \multicolumn{2}{c|}{$\mathrm{N}=253806$} \\
\hline
\end{tabular}

Table 5: Effects of obesity levels on the likelihood of good physical health.

\begin{tabular}{|c|c|c|c|c|c|c|c|}
\hline & \multirow{2}{*}{ B } & \multirow{2}{*}{ S.E. } & \multirow{2}{*}{ Wald } & \multirow{2}{*}{ Sig. } & \multirow{2}{*}{$\begin{array}{l}\text { Exp(B)(odds } \\
\text { ratio) }\end{array}$} & \multicolumn{2}{|c|}{ 95\% C.I.for EXP(B) } \\
\hline & & & & & & Lower & Upper \\
\hline \multicolumn{8}{|c|}{ Demographics } \\
\hline Female & -.166 & .013 & 164.380 & .000 & .847 & .826 & .869 \\
\hline Latino & -.063 & .021 & 9.125 & .003 & .939 & .902 & .978 \\
\hline Young & -.206 & .018 & 127.484 & .000 & .814 & .786 & .844 \\
\hline Veteran & .095 & .016 & 37.281 & .000 & 1.099 & 1.066 & 1.133 \\
\hline \multicolumn{8}{|c|}{ Household Climate } \\
\hline $\begin{array}{l}\text { Dependent } \\
\text { Children }\end{array}$ & -.066 & .005 & 164.335 & .000 & .936 & .927 & .946 \\
\hline $\begin{array}{l}\text { No Adult } \\
\text { Women }\end{array}$ & .044 & .017 & 6.657 & .010 & 1.045 & 1.011 & 1.080 \\
\hline No Adult Men & .166 & .013 & 169.507 & .000 & 1.180 & 1.151 & 1.210 \\
\hline \multicolumn{8}{|c|}{ Weight/Obesity } \\
\hline Underweight & -.181 & .038 & 23.010 & .000 & .835 & .775 & .899 \\
\hline Obese class 1 & -.076 & .012 & 39.561 & .000 & .927 & .905 & .949 \\
\hline Obese class 2 & -.135 & .018 & 53.246 & .000 & .874 & .843 & .906 \\
\hline Obese class 3 & -.190 & .024 & 62.006 & .000 & .827 & .789 & .867 \\
\hline \multicolumn{8}{|l|}{ SES } \\
\hline Unemployed & -.413 & .016 & 640.440 & .000 & .661 & .641 & .683 \\
\hline Education Level & .000 & .005 & .005 & .946 & 1.000 & .989 & 1.010 \\
\hline Income & .027 & .003 & 82.349 & .000 & 1.028 & 1.022 & 1.034 \\
\hline $\begin{array}{l}\text { Has personal } \\
\text { Cell phone }\end{array}$ & .040 & .013 & 10.268 & .001 & 1.041 & 1.016 & 1.067 \\
\hline \multicolumn{8}{|c|}{ Health Behavior } \\
\hline Non-Smoker & -.058 & .010 & 35.201 & .000 & .944 & .926 & .962 \\
\hline Current-drinker & .024 & .010 & 5.586 & .018 & 1.024 & 1.004 & 1.044 \\
\hline $\begin{array}{l}\text { Pneumonia } \\
\text { shot }\end{array}$ & -.031 & .010 & 8.886 & .003 & .969 & .950 & .989 \\
\hline Physical Ex. & .232 & .011 & 422.788 & .000 & 1.261 & 1.233 & 1.289 \\
\hline Seat belt always & .029 & .013 & 4.771 & .029 & 1.029 & 1.003 & 1.057 \\
\hline HIV-tested & -.139 & .011 & 158.617 & .000 & .870 & .851 & .889 \\
\hline \multicolumn{8}{|c|}{ Access to Care } \\
\hline Insured & -.135 & .019 & 53.189 & .000 & .874 & .842 & .906 \\
\hline $\begin{array}{l}\text { Has personal } \\
\text { doctor }\end{array}$ & -.176 & .017 & 111.818 & .000 & .839 & .812 & .867 \\
\hline Cost-barred & -.524 & .017 & 989.999 & .000 & .592 & .573 & .612 \\
\hline \multicolumn{8}{|c|}{ Health Capital } \\
\hline Heart Attack & -.180 & .021 & 70.323 & .000 & .835 & .801 & .871 \\
\hline Angina & -.245 & .021 & 139.793 & .000 & .783 & .751 & .815 \\
\hline Stroke & -.182 & .024 & 57.365 & .000 & .834 & .795 & .874 \\
\hline
\end{tabular}




\section{Current Research in Diabetes \& Obesity Journal}

\begin{tabular}{|c|c|c|c|c|c|c|c|}
\hline Asthma & -.336 & .015 & 528.756 & .000 & .714 & .694 & .735 \\
\hline Other cancer & -.216 & .015 & 202.005 & .000 & .806 & .782 & .830 \\
\hline COPD & -.433 & .018 & 552.713 & .000 & .649 & .626 & .673 \\
\hline Arthritis & -.408 & .010 & 1552.326 & .000 & .665 & .652 & .679 \\
\hline Depression & -.480 & .012 & 1509.852 & .000 & .619 & .604 & .634 \\
\hline Kidney & -.398 & .026 & 234.291 & .000 & .671 & .638 & .707 \\
\hline Poor Sight & -.298 & .013 & 563.869 & .000 & .742 & .724 & .761 \\
\hline Diabetes & -.222 & .014 & 244.823 & .000 & .801 & .379 & .358 \\
\hline Disability & -1.004 & .012 & 7127.997 & .000 & .366 & .375 \\
\hline $\begin{array}{c}\text { Assistive } \\
\text { Devices }\end{array}$ & -.579 & .017 & 1220.344 & .000 & .561 & .543 & .579 \\
\hline Model fit & $\mathrm{R} 2=.265$ & \multicolumn{2}{|c|}{$\chi 2=54950.66$} & \multicolumn{2}{|c|}{ Prediction $73 \%$} & & $\mathrm{~N}=254405$ \\
\hline
\end{tabular}

Likelihood of good general health Table 4 displays results of estimates of the likelihood of good general health and the effects of three obesity classes on the likelihood of good general health. Relative to the control group (normal- and overweight), all obesity levels have a negative and statistically significant $(\mathrm{P} \leq$ 0.000 ) effect on the likelihood of good general health. The Wald statistics of the three obesity classes indicate that the Class 3 $(B M I \geq 40)$ has the most significant negative effects while Class 1 has the lowest effects. The coefficients are: -.079 for Class 1 , -.258 for Class 2 and-.469 for Class 3 obesity. These numbers suggest that Class 3 obesity has almost six times greater negative effect on the likelihood of good general health than Class 1 and that Class 2 has more than three times greater effect than Class 1. These numbers indicate that the greater the obesity level, the greater the negative effect on the likelihood of good general health.

Other statistically significant negative predictors of the likelihood good general health include being Latino/a, underweight $(\mathrm{BMI}<18.5)$, unemployed, barriers to accessing health care and having poor health capital (i.e. having chronic health conditions). However, being female or young significantly and positively affect the likelihood of good general health. The same is true about being in a household with no adult males and having dependent children. Furthermore, the results indicate that some measure of good health behavior (not smoking, engaging in physical exercise and wearing seat belts) positively and significantly affects the likelihood of good general health. Conversely some indicators of health behaviors (HIV-testing, pneumonia shots) show negative effects.

The likelihood of good physical health Table 5 Physical health analysis results is similar to the general health results. They indicate that all three obesity classes negatively and significantly $(\mathrm{P} \leq 0.000)$ affect the likelihood of good physical health. The coefficients also indicate that the higher the obesity class, the greater the effects. The Wald statistics suggest that the higher the obesity class, the more significant the effects on the likelihood of good physical health. Other statistically significant negative predictors of the likelihood good physical health include being female, Latino/a, young and underweight
(BMI<18.5), unemployed, having dependent children, having barriers to accessing health care and having poor health capital stock (i.e. having chronic health conditions).

\section{Discussion}

The study results indicate significant health differences across the three obesity classes. The higher the obesity class, the lower the likelihood of good self-assessed general or physical health and the more the number of days the individuals experienced poor health. The higher the obesity class, the greater the proportions diagnosed with chronic health conditions except for skin cancer where the trend is opposite the higher the obesity class, the lower the proportions diagnosed with skin cancer. A possible explanation for this outcome could be that people with heavier weights are less likely to sunbathe than people with less bodyweight. With current emphasis and attention to evidence-based care and interventions, it is necessary to recognize that there are variations in obesity levels and in their effects on health and quality of life. It is important to gather detailed information about the different obesity classes and the different effects they have on health. Such information will provide means of creating more targeted interventions and treatments. Armed with detailed information and evidence about the different obesity levels, practitioners and policy makers can avoid painting obesity with a broad brush, which might create interventions that might not work for all obesity levels. For effective evidence-based interventions, it is necessary to decipher the varying effects of obesity on health conditions and to find out which conditions are affected by what obesity levels and how.

These findings indicate that the three obesity levels have different impacts on health. Individuals with Class 3 obesity experience about 4 days more of poor physical health, 3 days more of poor mental and general health per month that those with Class 1 obesity. The difference between Classes 1 and 2 is about one day more while between Classes 2 and 3 is about 2 days. Viewed in terms of current US average hourly earnings of $\$ 25.25$ [49], 4 days difference between obesity Classes 1 and 3 translates into $\$ 808$ earned per month, or $\$ 9,696$ per year- 


\section{Current Research in Diabetes \& Obesity Journal}

a significant difference. These numbers suggest significant differences in the impact of obesity classes on productivity.

Furthermore, after controlling for other factors that affect health, including demographics, household climate, SES, health behavior, access to care and individual health capital stock, relative to individuals with normal weight or those slightly overweight, those in the three obesity classes have lower likelihoods of experiencing good physical or good general health. An examination of the likelihood of good general health reveals that obesity Class 3 has close to six times the negative effects of Class 1, while Class 2 has three times the effects of Class 1. Obesity Class 3 has about two times the negative effects of Class 2 on the likelihood of good general health. Similarly, an examination of the likelihood of good physical health reveals that obesity Class 3 has 2.5 times the negative effects of Class 1 . Obesity Class 2 has 1.8 times the negative effects of Class 1 on the likelihood of good physical health.

\section{Conclusion}

Health effects of obesity vary by obesity class. These findings are significant even after controlling for other factors that affect health such as demographics, socioeconomic status, household climate, individual health behavior, access to health care and individual health capital stock. The effects of each obesity class are different. The pattern of obesity effects on physical and general health is different from the mental health. Given these differences and current emphasis on evidence-based interventions and treatments, it is important to examine obesity variations rather that viewing it as a single health condition. The obesity levels might require more targeted interventions rather than a single intervention for all three classes.

\section{References}

1. Finucane MM, Stevens GA, Cowan MJ, Danaei G, Lin JK, et al. (2011) National, regional, and global trends in body-mass index since 1980: systematic analysis of health examination surveys and epidemiological studies with 960 country-years and 9. 1 million participants. The Lancet 377(9765): 557-567.

2. Greenberg JA (2013) Obesity and early mortality in the United States. Obesity (Silver Spring) 21(2): 405-412

3. Finkelstein EA, Brown DS, Wrage LA, Allaire BT, Hoerger TJ, et al. (2010) Individual and aggregate years-of-life-lost associated with overweight and obesity. Obesity (Silver Spring) 18(2): 333-339.

4. Flegal KM, Graubard BI, Williamson DF, Gail MH (2005) Excess deaths associated with underweight, overweight, and obesity. JAMA 293(15): 1861-1867.

5. Ogden CL, Carroll M, Kit B, Flegal K (2013) Prevalence of obesity among adults: United States, 2011-2012. NCHS data brief 131: 1-8.

6. Muennig P, Lubetkin E, Jia H, Franks P (2006) Gender and the burden of disease attributable to obesity. Am J Public Health 96(9): 1662-1668.

7. Dietz WH (1998) Health consequences of obesity in youth: childhood predictors of adult disease. Pediatrics 101(3 Pt 2): 518-525.

8. Wabitsch M (2000) Overweight and obesity in European children: definition and diagnostic procedures, risk factors and consequences for later health outcome. Eur J Pediatr 159(Suppl 1): S8-13.
9. Hammond RA, Levine R (2010) The economic impact of obesity in the United States. Diabetes Metab Syndr Obes 3: 285-295.

10. Levi J, Segal LM, St. Laurent R, Kohn D (2011) F as in Fat: How Obesity Threatens America's Future. Trust for America's Health.

11. Ford ES, Williamson DF, Liu S (1997) Weight change and diabetes incidence: findings from a national cohort of US adults. Am J Epidemiol 146(3): 214-222.

12. Resnick HE, Valsania P, Halter JB, Lin X (2000) Relation of weight gain and weight loss on subsequent diabetes risk in overweight adults. J Epidemiol Community Health 54(8): 596-602.

13. Mokdad AH, Ford ES, Bowman BA, Dietz WH, Vinicor F, et al. (2003) Prevalence of obesity, diabetes, and obesity-related health risk factors, 2001. JAMA 289(1): 76-79.

14. Thomsen M, Nordestgaard BG (2014) Myocardial infarction and ischemic heart disease in overweight and obesity with and without metabolic syndrome. JAMA Intern Med 174(1): 15-22.

15. Cepeda-Valery B, Slipczuk L, Figueredo VM, Pressman GS, Morris DL, et al. (2013) Association between obesity and infarct size: insight into the obesity paradox. Int J Cardiol 167(2): 604-606.

16. Labounty TM, Gomez MJ, Achenbach S, Al-Mallah M, Berman DS, et al. (2013) Body mass index and the prevalence, severity, and risk of coronary artery disease: an international multicentre study of 13,874 patients. Eur Heart J Cardiovasc Imaging 14(5): 456-463.

17. Towfighi A, Zheng L, Ovbiagele B (2010) Weight of the obesity epidemic: rising stroke rates among middle-aged women in the United States. Stroke 41(7): 1371-1375.

18. Rhéaume C, Leblanc MÈ, Poirier P (2011) Adiposity assessment: explaining the association between obesity, hypertension and stroke. Expert Rev Cardiovasc Ther 9(12): 1557-1564.

19. Kernan WN, Inzucchi SE, Sawan C, Macko RF, Furie KL, et al. (2013) Obesity: a stubbornly obvious target for stroke prevention. Stroke 44(1): 278-286.

20. Holguin F, Bleecker ER, Busse WW (2011) Obesity and asthma: an association modified by age of asthma onset. J Allergy Clin Immunol 127(6): 1486-1493e1482.

21. Gruchala-Niedoszytko M, Malgorzewicz S, Niedoszytko M, Gnacinska M, Jassem E, et al. (2013) The influence of obesity on inflammation and clinical symptoms in asthma. Adv Med Sci 58(1): 15-21.

22. Mahadev S, Farah CS, King GG, Salome CM (2013) Obesity, expiratory flow limitation and asthma symptoms. Pulm Pharmacol Ther 26(4): 438-443.

23. Celedon JC, Kolls JK (2014) An innate link between obesity and asthma. Nat Med 20(1): 19-20.

24. Vozoris NT, O’Donnell DE (2012) Prevalence, risk factors, activity limitation and health care utilization of an obese, population-based sample with chronic obstructive pulmonary disease. Can Respir J 19(3): e18-24.

25. Guerra S, Sherrill DL, Bobadilla A, Martinez FD, Barbee RA, et al. (2002) The relation of body mass index to asthma, chronic bronchitis, and emphysema. Chest 122(4): 1256-1263.

26. Enzinger PC, Mayer RJ (2003) Esophageal cancer. N Engl J Med 349(23): 2241-2252.

27. Hursting SD, Lashinger LM, Wheatley KW, Rogers CJ, Colbert LH, et al. (2008) Reducing the weight of cancer: mechanistic targets for breaking the obesity-carcinogenesis link. Best Pract Res Clin Endocrinol Metab 22(4): 659-669.

28. Borgeson E, Sharma K (2013) Obesity, immunomodulation and chronic kidney disease. Curr Opin Pharmacol 13(4): 618-624. 


\section{Current Research in Diabetes \& Obesity Journal}

29. Chow WH, Gridley G, Fraumeni JF, Jarvholm B (2000) Obesity, hypertension, and the risk of kidney cancer in men. $\mathrm{N}$ Engl J Med 343(18): 1305-1311.

30. Giovannucci E (2007) Metabolic syndrome, hyperinsulinemia, and colon cancer: a review. Am J Clin Nutr 86(3): s836-s842.

31. Larsson SC, Wolk A (2007) Overweight, obesity and risk of liver cancer: a meta-analysis of cohort studies. Br J Cancer 97(7): 1005-1008.

32. Larsson SC, Wolk A (2007) Obesity and the risk of gallbladder cancer: a meta-analysis. Br J Cancer 96(9): 1457-1461.

33. Key TJ, Appleby PN, Reeves GK, Roddam A, Dorgan JF, et al. (2003) Body mass index, serum sex hormones, and breast cancer risk in postmenopausal women. J Natl Cancer Inst 95(16): 1218-1226.

34. Wolin KY, Carson K, Colditz GA (2010) Obesity and cancer. Oncologist 15(6): 556-565.

35. Calle EE, Rodriguez C, Walker-Thurmond K, Thun MJ, (2003) Overweight, obesity, and mortality from cancer in a prospectively studied cohort of US adults. N Engl J Med 348(17): 1625-1638.

36. Finkelstein EA, Khavjou OA, Thompson H, Trogdon JG, Pan L, et al (2012) Obesity and severe obesity forecasts through 2030. Am J Prev Med 42(6): 563-570.

37. Sturm R, Hattori A (2013) Morbid obesity rates continue to rise rapidly in the United States. Int J Obes (Lond.) 37(6): 889-891.

38. Sturm R (2003) Increases in clinically severe obesity in the United States, 1986-2000. Arch Intern Med 163(18): 2146-2148.

39. (2000) World Health Organization. Obesity: preventing and managing the global epidemic, pp. 252.

40. Grossman M (1972) On the concept of health capital and the demand for health. The Journal of Political Economy 80(2): 223-255.
41. Grossman M, Joyce T (1990) Unobservable, Pregnancy Resolutions, and Birth-Weight Production Functions in New York City. Journal of Political Economy 98(5): 983-1007.

42. Behrman JR, Rosenzweig MR (2004) Returns to birth weight. The Review of Economics and Statistics 86(2): 586-601.

43. Wehby GL, Murray JC, Castilla EE, Lopez-Camelo JS, Ohsfeldt RL, et al. (2009) Prenatal care effectiveness and utilization in Brazil. Health Policy Plan 24(3): 175-88.

44. Mityakov S Mroz T (2013) Economic theory as a guide for the specification and interpretation of empirical health production functions, pp. 1-81.

45. Rosenzweig MR, Schultz TP (1983) Estimating a Household Production Function: Heterogeneity, the Demand for Health Inputs, and Their Effects on Birth Weight. Journal of Political Economy 91(5): 723-746.

46. Conti G, Heckman JJ, Urzua S (2011) Early endowments, education and health. Human Capital and Economic Opportunity:

A Global Working Group, pp. 1-54.

47. Wehby GL, Prater K, McCarthy AM, Castilla EE, Murray JC, et al. (2011) The impact of maternal smoking during pregnancy on early child neurodevelopment. J Hum Cap 5(2): 207-254.

48. (2014) Centers for Disease Control \& Prevention, National Center for Chronic Disease Prevention and Health Promotion. About the Behavioral Risk Factor Surveillance System (BRFSS).

49. (2017) US Bureau of Labor Statistics, Average Hourly and Weekly Earnings of All Employees on Private Nonfarm Payrolls by Industry Sector, Seasonally Adjusted.

Your next submission with Juniper Publishers will reach you the below assets

- Quality Editorial service

- Swift Peer Review

- Reprints availability

- E-prints Service

- Manuscript Podcast for convenient understanding

- Global attainment for your research

- Manuscript accessibility in different formats ( Pdf, E-pub, Full Text, Audio)

- Unceasing customer service

Track the below URL for one-step submission https://juniperpublishers.com/online-submission.php 